
vibration reduction of a thick plate using singular value decomposition approach

\author{
Azin Nadi $^{\bowtie}$, Mojtaba Mahzoon \& Ehsan Azadi Yazdi \\ This paper uses the singular value decomposition approach to find the optimal distribution of a set of \\ piezoelectric actuators and sensors in order to suppress the vibrations of a thick plate. The dynamic \\ model of the system is derived using Mindlin plate theory and consequently, the finite difference \\ method is employed to divide the thick plate to a finite number of nodes with appropriate horizontal \\ and vertical distances. To compute the control force of piezoelectric actuators, the singular value \\ decomposition approach for the column control matrix is supposed as the fitness function of an \\ optimization problem. Through a genetic algorithm, the optimized solution is obtained. The results of \\ numerical simulations indicate the optimal location achieved by the proposed method outperforms \\ the previous results in suppressing the vibrations of a thick plate.
}

The problem of finding the optimal configuration of piezoelectric patches on a flexible plate for vibration suppression is one of the primary interests in engineering design. In smart structures, sensors and actuators work in a control system to achieve a higher performance in vibration suspension compared to the conventional passive systems. In such systems, piezoelectric sensors and actuators are widely used because they are inexpensive, reliable, fast, low weight, and low power ${ }^{1}$. Therefore, the optimal location of piezoelectric patches to improve controller optimality is one of the important open research problems. It is well known that misplaced patches (sensors and actuators) may lead to issues such as the lack of observability and controllability ${ }^{2}$.

Several studies have been conducted on the optimal position of piezoelectric actuators and sensors on thin plates by considering various objective functions and techniques. In terms of the objective function based on controllability and observability index, the optimal location of piezoelectric actuators on a composite structure by Padoin et al. ${ }^{3}$ and a thin plate using the Modified Control Matrix and Singular Value Decomposition (MCSVD) technique by Chhabra et al. ${ }^{1}$ was suggested. Moreover, the optimal position and the number of active elements for separate sensors and actuators were found by Bruant et al. ${ }^{4}$ and Chakraborty et al. ${ }^{5}$ Biglar et al. ${ }^{6}$ suggested an objective function based on the spatial controllability or observability Gramian and the incorporation of residual modes to decrease the spillover effect for the active vibration control of a plate to obtain optimal orientations and positions of piezoelectric patches. The optimal location of piezoelectric patches has been optimized with the objective function based on the maximization of Gramian matrix visibility and distributing the amount of strain energy on the plate by Loghmani et al. ${ }^{7}$, Ferrari and Amabili ${ }^{8}$, respectively. In addition, the $\mathrm{H} \infty$ norm of the closed-loop system as the objective function was considered in ${ }^{9}$. Active vibration suppression of a flexible structure bonded with optimized piezoelectric pairs was developed by Daraji and Hale ${ }^{10}$, and reduction of the genetic algorithm search space by designing a conditional filter was proposed in ${ }^{11}$. In other researches, the optimal location of sensors in structural health monitoring systems was studied to cover a specific number of low-frequency modes $^{12}$. In large-scale structures due to moving loads, an approach based on convex relaxation was proposed by Błachowski et al. ${ }^{13}$ to efficiently optimize sensor layout. Also, the optimal places of triaxial accelerometers were presented based on Effective Independence by Kammer and Tinker ${ }^{14}$.

Plates are widely used as structural elements in modern construction aerospace, engineering, and aeronautical industries. Several models for the plates based on various theories were studied in the literature. The deflection of the Kirchhoff plate was first approximated with deep physical informed feedforward neural networks by 


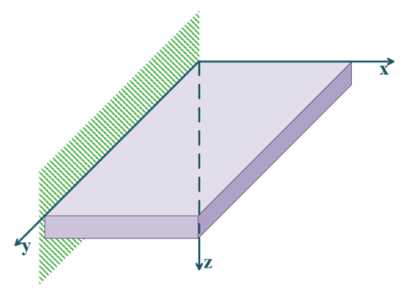

Figure 1. A cantilever thick plate.

minimizing a loss function related to avoiding a FEM discretization entirely in ${ }^{15}$. The proposed deep collocation and energy methods with a set of DNNs approximating the transversal deflection are proven to be effective in the bending analysis of the Kirchhoff plate ${ }^{16}$. The Mindlin plate theory is used to obtain the dynamic model of a thick plate ${ }^{17}$. Moreover, a three-dimensional piezoelectric structure was extended to the detection of inclusions by Nanthakumar et al. ${ }^{18}$ for inverse problems.

On the one hand, the majority of studies in the area of the active vibration suppression of plates and shells by optimal location of piezoelectric patches (actuators or sensors) have selected the number of patches arbitrarily and optimized just their location on a thin plate. However, this causes weak vibration suppression or high added weight, costs and energy requirement. Moreover, although many researches have been done regarding the active vibration control of smart structures, there are still scopes and needs to be improved. Moreover, the problem of optimal configuration of piezoelectric patches on flexible a structure where improved performance and efficiency is one of the most interesting issues among engineering design.

If the effects of transverse shear deformation do not consider in relatively thick plates, it causes error in the calculation of deflections and stress resultants of such plates. Because, thick plates are main members of various structures. They can be applied as elements of engine foundations, reinforced concrete bridges, parts of various floating structures ${ }^{19,20}$. In addition, it is uneconomical to implement the cost of sensors and actuators into account to mount patches on every part of a structure. Moreover, the weight is a vital factor in various industry especially aeronautical. The resulting configuration and number of sensors and actuators must be optimal. A contribution of the present paper is related to find a new objective function based on the optimum number of patches as well as their optimal location on the thick plate for vibration reduction by using a binary-coded genetic algorithm that modified by adding a feasibility test in the mutation step.

In this study, a finite difference model of a thick plate with piezoelectric sensors and actuators has been developed, and Linear Quadratic Regulator (LQR) optimal control scheme has been used to study the effectiveness of the controller. The results of the present research have raised the closed-loop average $\mathrm{dB}$ gain as compared to the optimal position of random selection, the norm of singular value decomposition, and using optimal placement which is obtained on a thin plate with the same number of patches.

The method has been used in the present study has the following advantages compared to the previous articles:

1. Instead of using the thin plate $i^{21}$ for finding the optimal location of piezoelectric patches, in this paper, the optimization of piezoelectric patches locations on a cantilever thick plate based on Mindlin plate theory has been carried out.

2. Instead of selecting a specified number of piezoelectric patches on the thin plate in ${ }^{1}$, in this paper, the optimization of the number of piezoelectric patches on a cantilever thick plate has been performed.

3. Although the method presents in this paper for obtaining the fitness function somewhat similar to the method presented in $^{1}$, this paper considers the optimal number of piezoelectric patches by using the Mindlin plate theory for modeling the cantilever thick plate. Moreover, the objective is to attenuate the vibrations by considering the modified control matrix and singular value decomposition and developing a cost function using a binary-coded genetic algorithm.

The paper is arranged as follow:

In "Thick plate formulation" section to "State-space model" section, the equation of motion of the thick plate with piezoelectric patches are solved through the FDM by applying the Mindlin plate theory and the LQR optimal control theory. In "Singular value decomposition (SVD)" section, Singular Value Decomposition has been discussed for finding a fitness function which is used in genetic algorithm. Then, we discuss the 1-bit coding of the piezoelectric actuators and sensors installed on the thick plate. The binary-coded genetic algorithm (GA) is employed to find the optimal placement and number of the patches on the cantilever thick plate in "1-bit coding plate by genetic algorithm" section. Moreover, average closed-loop dB gain reduction has been applied to validate the optimal placements of piezoelectric actuators and sensors obtained in "Average closed-loop $\mathrm{dB}$ gain reduction" section. Finally, the results and conclusion are presented in "Results and discussion" and "Conclusions" sections, respectively.

\section{Formulation and methodology}

Thick plate formulation. Figure 1 shows the coordinate axes and boundary conditions of a thick plate.

The equation of motion for a rectangular Mindlin plate under time-varying dynamic load as a function $P(x, y, t)$ is (see Supplementary Note for detailed derivations): 


$$
\left[D \nabla^{4} w_{b}-J\left(1+\frac{\bar{m} D}{J S}\right) \frac{\partial^{2}}{\partial t^{2}} \nabla^{2} w_{b}+\bar{m} \frac{\partial^{2}}{\partial t^{2}}\left(w_{b}+\frac{J}{S} \frac{\partial^{2} w_{b}}{\partial t^{2}}\right)\right]=P(x, y, t)
$$

where $w_{b}$ is bending displacement of thick plate. $\bar{m}=\rho h$ is the mass per the unit area, $\rho$ and $h$ are density of the plate and plate thickness, respectively. $J=\rho I=\rho\left(\frac{h^{3}}{12}\right)$ is mass inertia moment of plate cross-section. $D=\frac{E h^{3}}{12\left(1-v^{2}\right)}$ and $S=k G h$ are the bending and shear strength of the plate, respectively. $E, G, v, k$ are Young's modulus, Shear modulus, Poisson's ratio, shear correction coefficient, respectively. $\nabla^{2}(\cdot)=\frac{\partial^{2}(\cdot)}{\partial x^{2}}+\frac{\partial^{2}(\cdot)}{\partial y^{2}}$ is the Laplace differential operator. $\nabla^{4}$ is a differential operator equivalent to $\frac{\partial^{4}}{\partial x^{4}}+2 \frac{\partial^{4}}{\partial x^{2} \partial y^{2}}+\frac{\partial^{4}}{\partial y^{4}}$.

Equation (1) can be rewritten based on internal moments.

$$
\left[\frac{\partial^{2} M_{x}}{\partial x^{2}}+2 \frac{\partial^{2} M_{x y}}{\partial x \partial y}+\frac{\partial^{2} M_{y}}{\partial y^{2}}-\frac{J}{D(1+v)}\left(1+\frac{\bar{m} D}{J S}\right) \frac{\partial^{2}}{\partial t^{2}}\left(M_{x}+M_{y}\right)-\bar{m} \frac{\partial^{2} w_{b}}{\partial t^{2}}-\frac{\bar{m} J}{S} \frac{\partial^{4} w_{b}}{\partial t^{4}}\right]=-P(x, y, t)
$$

$M_{x}, M_{y}$ and $M_{x y}$ are the internal moments of the thick plate.

If $\mathrm{N}$ piezoelectric actuators are fully connected to the thick plate. The patches will generate moments which effect on the internal moments of the thick plate. Because piezoelectric patches are usually lighter and smaller than the host plate, the effect of their mass on dynamics of the plate can be ignored. Therefore, the above equations are modified as follows:

$$
\begin{aligned}
& {\left[\frac{\partial^{2}\left(M_{x}-m_{p x}\right)}{\partial x^{2}}+2 \frac{\partial^{2}\left(M_{x y}-m_{p x y}\right)}{\partial x \partial y}+\frac{\partial^{2}\left(M_{y}-m_{p y}\right)}{\partial y^{2}}-\frac{J}{D(1+v)}\right.} \\
& \left.\left(1+\frac{\bar{m} D}{J S}\right) \frac{\partial^{2}}{\partial t^{2}}\left(M_{x}-m_{p x}+M_{y}-m_{p y}\right)-\bar{m} \frac{\partial^{2} w_{b}}{\partial t^{2}}-\frac{\bar{m} J}{S} \frac{\partial^{4} w_{b}}{\partial t^{4}}\right]=-P(x, y, t)
\end{aligned}
$$

where $m_{p x}, m_{p y}$ and $m_{p x y}$ indicate the bending and torsional moment created by the piezoelectric actuators. Equation (3) can be rewritten as Eq. (4).

$$
\begin{aligned}
& {\left[D \nabla^{4} w_{b}-J\left(1+\frac{\bar{m} D}{J S}\right) \frac{\partial^{2}}{\partial t^{2}}\left(\frac{\partial^{2} w_{b}}{\partial x^{2}}+\frac{\partial^{2} w_{b}}{\partial y^{2}}\right)+\bar{m} \frac{\partial^{2} w_{b}}{\partial t^{2}}+\frac{\bar{m} J}{S} \frac{\partial^{4} w_{b}}{\partial t^{4}}\right]} \\
& \quad=\left[P(x, y, t)-\frac{\partial^{2} m_{p x}}{\partial x^{2}}-2 \frac{\partial^{2} m_{p x y}}{\partial x \partial y}-\frac{\partial^{2} m_{p y}}{\partial y^{2}}+\frac{J}{D(1+v)}\left(1+\frac{\bar{m} D}{J S}\right) \frac{\partial^{2}}{\partial t^{2}}\left(m_{p x}+m_{p y}\right)\right] .
\end{aligned}
$$

The moments generated by the $\mathrm{N}$ piezoelectric actuators have the same thickness and properties subjected to a voltage can be calculated using Eq. $(5)^{22}$.

$$
\begin{gathered}
\left\{\begin{array}{c}
m_{p x}(x, y, t)=C_{0} \frac{d_{31}}{h_{P Z T}} V(t) R_{p}^{n_{a}}(x, y) \\
m_{p y}(x, y, t)=C_{0} \frac{d_{32}}{h_{P Z T}} V(t) R_{p}^{n_{a}}(x, y) \\
m_{p x y}(x, y, t)=C_{0} \frac{d_{36}}{h_{P Z T}} V(t) R_{p}^{n_{a}}(x, y)=0
\end{array}\right. \\
R_{p}^{n_{a}}(x, y)=\left[H\left(x-x_{1}^{n_{a}}\right)-H\left(x-x_{2}^{n_{a}}\right)\right]\left[H\left(y-y_{1}^{n_{a}}\right)-H\left(y-y_{2}^{n_{a}}\right)\right]
\end{gathered}
$$

where $d_{31}, d_{32}$ and $d_{36}=0$ are the strain constants of the piezoelectric materials, $h_{P Z T}$ is patch thickness, $H($. is Heaviside function, $x_{1}^{n_{a}}$ and $y_{1}^{n_{a}}$ indicate the coordinates of the bottom left corner of $n_{a}$ th Piezoelectric patch, $x_{2}^{n_{a}}$ and $y_{2}^{n_{a}}$ indicate the coordinates of the top right corner of $n_{a}$ th Piezoelectric patch, $V(t)$ is the voltage, and $C_{0}$ is obtained from the relation $(7)^{22}$.

$$
C_{0}=\frac{-1}{6} \frac{1+v_{P Z T}}{1-v} \frac{E h^{2} K}{1+v-\left(1+v_{P Z T}\right) K}
$$

where $v$ and $v_{P Z T}$ are Poisson ratio of plate materials and piezoelectric materials, respectively. $K$ is given by ${ }^{22}$,

$$
K=\frac{-E_{P Z T}}{E} \frac{1-v^{2}}{1-v_{P Z T}^{2}} \frac{1.5 h_{P Z T} h\left(h+h_{P Z T}\right)}{2\left(0.125 h^{3}+h_{P Z T}^{3}\right)+1.5 h \times h_{P Z T}^{2}}
$$

where $E_{P Z T}$ is the modulus of elasticity piezoelectric patches.

Finite difference method. The first stage in using the finite difference method is dividing the thick plate to a finite mesh of nodes with the horizontal and vertical distance between each node shown by $\mathrm{H}$ and $\mathrm{L}$, respectively ${ }^{22}$. Also, additional knots outside the plate are used to show the boundary conditions on the cantilever thick plate in the finite difference method as shown in Fig. 2. In the next step, the nodes are divided to six sets (Fig. 3). Each set is determined by some equations explained in the following sections ${ }^{22}$. 


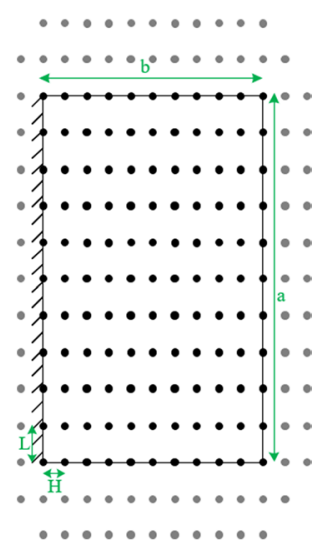

Figure 2. Original and additional nodes are represented by black and gray color, respectively.

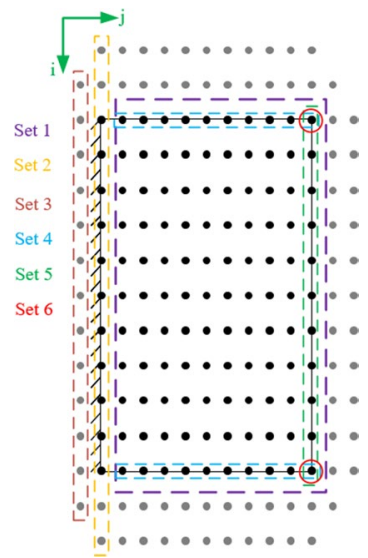

Figure 3. Node sets which include some nodes to assign by a number of equations in finite difference method.

Set 1 nodes. Each node in this set is represented by a relation obtained by Eq. (4), and terms related to the finite difference method in this equation are obtained from the following relationships.

$$
\begin{aligned}
& \nabla^{4} w_{b}(i, j, t) \cong\left[\left[\frac{6}{H^{4}}+\frac{6}{L^{4}}+\frac{8}{H^{2} L^{2}}\right] w_{b}(i, j, t)+\left[\frac{-4}{H^{4}}-\frac{-4}{H^{2} L^{2}}\right]\left(\begin{array}{c}
w_{b}(i, j-1, t)+w_{b}(i, j+1, t) \\
+w_{b}(i-1, j, t)+w_{b}(i+1, j, t)
\end{array}\right)\right. \\
& \left.+\frac{2}{H^{2} L^{2}}\left(\begin{array}{c}
w_{b}(i-1, j-1, t)+w_{b}(i-1, j+1, t) \\
+w_{b}(i+1, j-1, t)+w_{b}(i+1, j+1, t)
\end{array}\right)+\frac{1}{H^{4}}\left(\begin{array}{c}
w_{b}(i, j+2, t)+w_{b}(i, j-2, t) \\
+w_{b}(i-2, j, t)+w_{b}(i+2, j, t)
\end{array}\right)\right] \\
& \frac{\partial^{2} w_{b}(i, j, t)}{\partial x^{2}}=-\frac{1}{H^{2}}\left[w_{b}(i, j-1, t)-2 w_{b}(i, j, t)+w_{b}(i, j+1, t)\right] \\
& \frac{\partial^{2} w_{b}(i, j, t)}{\partial y^{2}}=-\frac{1}{L^{2}}\left[w_{b}(i-1, j, t)-2 w_{b}(i, j, t)+w_{b}(i+1, j, t)\right] \\
& \frac{\partial^{2} m_{p x}(i, j, t)}{\partial x^{2}} \cong \frac{-C_{0} d_{31}}{h_{P Z T} H^{2}} V(t)\left[R^{n_{a}}(i, j-1)-2 R^{n_{a}}(i, j)+R^{n_{a}}(i, j+1)\right] \\
& \frac{\partial^{2} m_{p y}(i, j, t)}{\partial y^{2}} \cong \frac{-C_{0} d_{32}}{h_{P Z T} L^{2}} V(t)\left[R^{n_{a}}(i-1, j)-2 R^{n_{a}}(i, j)+R^{n_{a}}(i+1, j)\right] \\
& \frac{\partial^{2} m_{p x y}(i, j, t)}{\partial x \partial y} \cong 0
\end{aligned}
$$


$i$ and $j$ represent the row and column of the node, respectively, and $t$ denotes time. By substituting relationships (9)-(14) in the relationship (4).

The time-varying dynamic load can be written as $P(x, y, t)=F(t) P(x, y)$ by the separation of variables.

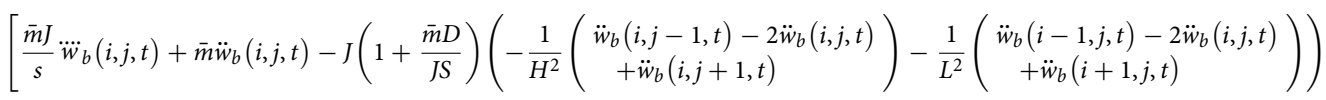

$$
\begin{aligned}
& \left.+D\left(\begin{array}{c}
{\left[\frac{6}{H^{4}}+\frac{6}{L^{4}}+\frac{8}{H^{2} L^{2}}\right] w_{b}(i, j, t)+\left[\frac{-4}{H^{4}}-\frac{-4}{H^{2} L^{2}}\right]\left(\begin{array}{c}
w_{b}(i, j-1, t)+w_{b}(i, j+1, t) \\
+w_{b}(i-1, j, t)+w_{b}(i+1, j, t)
\end{array}\right)} \\
+\frac{2}{H^{2} L^{2}}\left(\begin{array}{c}
w_{b}(i-1, j-1, t)+w_{b}(i-1, j+1, t) \\
+w_{b}(i+1, j-1, t)+w_{b}(i+1, j+1, t)
\end{array}\right)+\frac{1}{H^{4}}\left(\begin{array}{c}
w_{b}(i, j+2, t)+w_{b}(i, j-2, t) \\
+w_{b}(i-2, j, t)+w_{b}(i+2, j, t)
\end{array}\right)
\end{array}\right)\right] \\
& =F(t) P(i, j)-V(t) Z^{n_{a}}(i, j) \\
& Z^{n_{a}}(i, j)=\frac{-C_{0} d_{31}}{h_{P Z T} H^{2}}\left[R_{p}^{n_{a}}(i, j-1)-2 R_{p}^{n_{a}}(i, j)+R_{p}^{n_{a}}(i, j+1)\right]+\frac{-C_{0} d_{32}}{h_{P Z T} L^{2}}\left[R_{p}^{n_{a}}(i-1, j)-2 R_{p}^{n_{a}}(i, j)+R_{p}^{n_{a}}(i+1, j)\right]
\end{aligned}
$$

Set 2 nodes. The set of second nodes is related to the clamped edge of the thick plate. The relationship of each node in this set is calculated according to the displacement at the clamped edge ${ }^{22}$.

$$
w_{b}(i, j, t)=0
$$

Set 3 nodes. The third nodes are the set of additional nodes on the left side of the clamped edge of the thick plate. To get the relationship of these nodes, the rotation around the clamped edge in boundary conditions is considered. Therefore, the relation of each node in this set is as follows ${ }^{22}$.

$$
w_{b}(i, j, t)=w_{b}(i, j+2, t)
$$

Set 4 nodes. The fourth node set includes free nodes at the bottom and top edges of the plate. The boundary conditions at these edges are as follows ${ }^{22}$.

$$
\left\{\begin{array}{c}
M_{y}(x, y, t)=-D\left[\frac{\partial^{2} w_{b}(x, y, t)}{\partial y^{2}}+v \frac{\partial^{2} w_{b}(x, y, t)}{\partial x^{2}}\right]=0 \\
\bar{Q}_{y}(x, y, t)=Q_{y}+\frac{\partial M_{x y}}{\partial x}=-D\left[\begin{array}{c}
\frac{\partial^{3} w_{b}(x, y, t)}{\partial y^{3}}+ \\
(2-v) \frac{\partial^{3} w_{b}(x, y, t)}{\partial x^{2} \partial y}
\end{array}\right]=0
\end{array}\right.
$$

where $\bar{Q}_{y}$ is the shearing force which includes the effect of torsional moment and the transverse shear force. $Q_{y}$ is transverse shear force (see Supplementary Note).

Therefore, the following two equations must be satisfied for each node in this set.

$$
\begin{aligned}
& {\left[(-2-2 v) w_{b}(i, j, t)+v\left[w_{b}(i, j-1, t)+w_{b}(i, j+1, t)\right]+w_{b}(i-1, j, t)+w_{b}(i+1, j, t)\right]=0} \\
& {\left[( 2 v - 6 ) \left[w_{b}(i-1, j, t)-w_{b}(i+1, j, t]+(2-v)\left[\begin{array}{c}
w_{b}(i-1, j-1, t)+w_{b}(i-1, j+1, t) \\
-w_{b}(i+1, j-1, t)-w_{b}(i+1, j+1, t)
\end{array}\right]\right.\right.} \\
& \left.\quad+w_{b}(i-2, j, t)-w_{b}(i+2, j, t)\right]=0
\end{aligned}
$$

Set 5 nodes. The fifth node set includes the free edge nodes to the right of the plate. The boundary conditions at these edges are as follows 22 .

$$
\left\{\begin{array}{c}
M_{x}(x, y, t)=-D\left[\frac{\partial^{2} w_{b}(x, y, t)}{\partial x^{2}}+v \frac{\partial^{2} w_{b}(x, y, t)}{\partial y^{2}}\right]=0 \\
\bar{Q}_{x}(x, y, t)=Q_{x}+\frac{\partial M_{x y}}{\partial y}=-D\left[\begin{array}{c}
\frac{\partial^{3} w_{b}(x, y, t)}{\partial x^{3}}+ \\
(2-v) \frac{\partial^{3} w_{b}(x, y, t)}{\partial x \partial y^{2}}
\end{array}\right]=0
\end{array}\right.
$$

where $\bar{Q}_{x}$ is the shearing force, including the effect of torsional moment and the transverse shear force. $Q_{x}$ is transverse shear force (see Supplementary Note).

Therefore, the following two equations must be satisfied for each node in this set.

$$
\left[(-2-2 v) w_{b}(i, j, t)+v\left[w_{b}(i-1, j, t)+w_{b}(i+1, j, t)\right]+w_{b}(i, j-1, t)+w_{b}(i, j+1, t)\right]=0 .
$$




$$
\begin{aligned}
& {\left[( 2 v - 6 ) \left[w_{b}(i, j+1, t)-w_{b}(i, j-1, t]+(2-v)\left[\begin{array}{c}
w_{b}(i-1, j+1, t)+w_{b}(i+1, j+1, t) \\
-w_{b}(i-1, j-1, t)-w_{b}(i+1, j-1, t)
\end{array}\right]\right.\right.} \\
& \left.\quad+w_{b}(i, j+2, t)-w_{b}(i, j-2, t)\right]=0
\end{aligned}
$$

Set 6 nodes. Finally, the sixth node set consists of two nodes in the corners of the free edge of the plate that must satisfy the following equation ${ }^{22}$.

$$
R_{c f}=2 D(1-v) \frac{\partial^{2} w_{b}(x, y, t)}{\partial x \partial y}=0
$$

where $R_{c f}$ is the concentrated forces. The relationship of the finite difference is as follows.

$$
\left[w_{b}(i-1, j+1, t)-w_{b}(i-1, j-1, t)+w_{b}(i+1, j-1, t)+w_{b}(i+1, j+1, t)\right]=0
$$

State-space model. Each node in the first set is denoted by the index from 1 to $\mathrm{n}, \mathrm{n}$ is the summation of the nodes in the first set. By getting $R_{p}^{n_{a}}(x, y)$ from the relation (6) and substituting it into relation (16) the value of $Z^{n_{a}}$ is obtained for each piezoelectric patch. The motion of each node in this set is calculated from Eq. (15), and it is related to mesh size, applied load, displacement of adjacent nodes, the properties of the thick plate, applied voltage by piezoelectric patches and $Z^{n_{a}}$ value in these nodes and adjacent node.

In addition, nodes in sets 2-6 are identified by indices from $n+1$ to $n_{t} . n_{t}$ is the sum of all nodes. The displacement of each node in this set is obtained according to the relationships mentioned in the previous sections. This method leads to a linear system as follows.

Where 0 and $I$ show the zero and identity matrices, respectively. $n_{a d}=n_{t}-n$ is the number of additional nodes $(\mathrm{ad})^{22}$. The variables $A M$ can be calculated from Eq. (15) for each node. The variables $A C 11, A C 12, A C 21$, and $A C 22$ are coefficients of $w$ in the sets 1-6.

The condensed matrix can be calculated by removing the extra nodes. The reduced system is as follows ${ }^{20}$.

$$
\begin{aligned}
& {\left[\begin{array}{cc}
\left(\frac{\bar{m} J}{s}\right) I_{(n \times n)} & 0_{\left(n \times n_{a d}\right)} \\
0_{\left(n_{a d} \times n\right)} & 0_{\left(n_{a d} \times n_{a d}\right)}
\end{array}\right]\left[\begin{array}{c}
\dddot{w}_{1} \\
\dddot{w}_{2} \\
\vdots \\
\dddot{w}_{n} \\
\dddot{w}_{n+1} \\
\vdots \\
\dddot{w}_{n_{t}}
\end{array}\right]+\left[\begin{array}{cc}
A M_{(n \times n)} & 0_{\left(n \times n_{a d}\right)} \\
0_{\left(n_{a d} \times n\right)} & 0_{\left(n_{a d} \times n_{a d}\right)}
\end{array}\right]\left[\begin{array}{c}
\ddot{w}_{1} \\
\ddot{w}_{2} \\
\vdots \\
\ddot{w}_{n} \\
\ddot{w}_{n+1} \\
\vdots \\
\ddot{w}_{n_{t}}
\end{array}\right]} \\
& +\left[\begin{array}{cc}
A C 11_{(n \times n)} & A C 12_{\left(n \times n_{a d}\right)} \\
A C 21_{\left(n_{a d} \times n\right)} & A C 22_{\left(n_{a d} \times n_{a d}\right)}
\end{array}\right]\left[\begin{array}{c}
w_{1} \\
w_{2} \\
\vdots \\
w_{n} \\
w_{n+1} \\
\vdots \\
w_{n_{t}}
\end{array}\right]=F(t)\left[\begin{array}{c}
P_{1} \\
P_{2} \\
\vdots \\
P_{n} \\
0 \\
\vdots \\
0
\end{array}\right]-V(t)\left[\begin{array}{ccc}
Z_{1}^{1} & \cdots & Z_{1}^{n_{a}} \\
Z_{2}^{1} & \cdots & Z_{2}^{n_{a}} \\
\vdots & \cdots & \vdots \\
Z_{n}^{1} & \cdots & Z_{n}^{n_{a}} \\
0 & \cdots & 0 \\
\vdots & \cdots & \vdots \\
0 & \cdots & 0
\end{array}\right] \\
& T=\left[\begin{array}{c}
I_{(n \times n)} \\
-A C 22^{-1} \times A C 21
\end{array}\right] \\
& A C_{n \times n}=T^{T} \times\left[\begin{array}{cc}
A C 11_{(n \times n)} & A C 12_{\left(n \times n_{a d}\right)} \\
A C 21_{\left(n_{a d} \times n\right)} & A C 22_{\left(n_{a d} \times n_{a d}\right)}
\end{array}\right] \times T \\
& \left(\frac{\bar{m} J}{s}\right)_{(n \times n)}\left[\begin{array}{c}
\dddot{w}_{1} \\
\dddot{w}_{2} \\
\vdots \\
\dddot{w}_{n}
\end{array}\right]+A M_{(n \times n)}\left[\begin{array}{c}
\ddot{w}_{1} \\
\ddot{w}_{2} \\
\vdots \\
\ddot{w}_{n}
\end{array}\right]+A C_{(n \times n)}\left[\begin{array}{c}
w_{1} \\
w_{2} \\
\vdots \\
w_{n}
\end{array}\right]=F(t)\left[\begin{array}{c}
P_{1} \\
P_{2} \\
\vdots \\
P_{n}
\end{array}\right]-V(t)\left[\begin{array}{ccc}
Z_{1}^{1} & \cdots & Z_{1}^{n_{a}} \\
Z_{2}^{1} & \cdots & Z_{2}^{n_{a}} \\
\vdots & \cdots & \vdots \\
Z_{n}^{1} & \cdots & Z_{n}^{n_{a}}
\end{array}\right]
\end{aligned}
$$

Assuming state variables as $x(t)=\left[w_{1}, \ldots, w_{n}, \dot{w}_{1}, \ldots, \dot{w}_{n}, \ddot{w}_{1}, \ldots, \ddot{w}_{n}, \dddot{w}_{1}, \ldots, \dddot{w}_{n}\right]^{T}$, the state space form is calculated as follows.

$$
\begin{aligned}
& \dot{x}(t)=A_{s} x(t)+B_{s}\{F(t) P-V(t) Z\} \\
& y(t)=C_{s} x(t)+D_{s}\{F(t) P-V(t) Z\}
\end{aligned}
$$

where 


$$
A_{s}=\left[\begin{array}{cccc}
0_{n} & I_{n} & 0_{n} & 0_{n} \\
0_{n} & 0_{n} & I_{n} & 0_{n} \\
0_{n} & 0_{n} & 0_{n} & I_{n} \\
-\left(\frac{s}{\bar{m} J}\right) A C & 0_{n} & -\left(\frac{s}{\bar{m} J}\right) A M & 0_{n}
\end{array}\right], B_{s}=\left[\begin{array}{c}
0_{n} \\
0_{n} \\
0_{n} \\
\left(\frac{s}{\bar{m} J}\right)
\end{array}\right], C_{s}=\left[\begin{array}{llll}
0_{n} & I_{n} & 0_{n} & 0_{n}
\end{array}\right], D_{s}=\left[0_{n}\right]
$$

The control force from actuators is calculated by the control matrix $B_{c}=B_{s} Z$, and $u=V(t)$ is control voltage of actuators $V(t)=\left[\begin{array}{llll}V_{1} & V_{2} & \cdots & V_{n_{a}}\end{array}\right]^{T}$.

On the other hand, the piezoelectric sensor voltage is equal to ${ }^{23}$.

$$
V_{s}(t)=\mathrm{R}_{p} r^{n_{a}} \int_{y_{1}^{n_{a}}}^{y_{2}^{n_{a}}} \int_{x_{1}^{n_{a}}}^{x_{2}^{n_{a}}}\left(e_{31} \frac{\partial^{2} \dot{w}}{\partial x^{2}}+e_{32} \frac{\partial^{2} \dot{w}}{\partial y^{2}}+2 e_{36} \frac{\partial^{2} \dot{w}}{\partial x \partial y}\right) d x d y
$$

where $\mathrm{R}_{p}$ and $r^{n_{a}}$ are the constant of the current amplifier and the distance between the middle plans of the plate and the sensor, respectively. $e_{31}, e_{32}$ and $e_{36}=0$ are the piezoelectric material stress constants of the piezoelectric patches $^{23}$. By considering the same mesh as mentioned before and applying finite difference method, Eq. (33) can be rewritten as

$$
V_{s}^{n_{a}}(t) \cong \mathrm{R}_{p} r^{n_{a}} \int_{y_{1}^{n_{a}}}^{y_{2}^{n_{a}}} \int_{1}^{n_{a}} x_{a}^{n_{a}}\left\{\frac{e_{31}}{H^{2}}[\dot{w}(i, j-1, t)-2 \dot{w}(i, j, t)+\dot{w}(i, j+1, t)]+e_{32}[\dot{w}(i-1, j, t)-2 \dot{w}(i, j, t)+\dot{w}(i+1, j, t)]\right\} d x d y
$$

Devoting the nodes from 1 to $\mathrm{n}$ can be simplified the Eq. (34).

$$
V_{s}^{n_{a}}(t) \cong \mathrm{R}_{p} r^{n_{a}} N_{\left(n_{a} \times n\right)} S_{(n \times n)}\left[\begin{array}{llll}
\dot{w}_{1} & \dot{w}_{2} & \cdots & \dot{w}_{n}
\end{array}\right]^{T}
$$

where $S$ is the coefficients calculated by finite difference method. The constant matrix $N$ indicates the weights related to the numerical integration for the elements which are covered by piezoelectric sensors, and the other elements are zeros.

The modified state-space output $y_{V}$ can be rewritten as

$$
y_{V}(t)=C_{c} x(t)+D_{s}\{F(t) P-V(t) Z\}
$$

where $C_{c}=\mathrm{R}_{p} r^{n_{a}} N_{\left(n_{a} \times n\right)} S_{(n \times n)} C_{s}$.

The active control gain can be determined by using LQR optimal control theory. Minimization of the performance index $J$ used for finding the optimal placements and optimal numbers.

$$
J=\frac{1}{2} \int_{0}^{\infty}\left(\{x\}^{T}[Q]\{x\}+\{u\}^{T}[R]\{u\}\right) d t
$$

The dimensions of the matrices $[Q]$ and $[R]$ are equal to the number of state variables and piezoelectric actuators, respectively.

The control law is given by assuming full state feedback.

$$
\{u\}=-[K]\{x\}
$$

with constant control gain

$$
[K]=[R]^{-1}\left[B_{c}\right]^{T}[S]
$$

By solving the Riccati equation given by below equation, $[S]$ can be calculated.

$$
\left[A_{s}\right]^{T}[S]+[S]\left[A_{s}\right]+[Q]-[S]\left[B_{c}\right][R]^{-1}\left[B_{c}\right]^{T}[S]=0
$$

The system will be stable when $[S]$ is positive definite.

Singular value decomposition (SVD). The SVD can factorize any matrix $B_{c}$ into three matrices. A diagonal matrix $\sigma$ with the same dimension as $B_{c}$ whose diagonal elements are the singular value of $B_{c}$ in a descending order. $M$ and $N$ are both unitary matrices $\left(M^{T} M=I \text { and } N N^{T}=I\right)^{24}$.

$$
B_{c}=M \sigma N^{T}
$$

Wang and Wang ${ }^{24}$ presented a controllability index for finding the optimal location of actuators by maximizing the global control force. Singular values can be considered as controllability index which shows the amount of control energy provided by the piezoelectric actuators on the plate for a certain control input. $\sigma_{k}$ is referred to as the kth degree of controllability of the system. The size and location of the piezoelectric actuators can be found by the magnitude of $\sigma_{k}$. A controllability index $\Omega$ is ${ }^{24}$ :

$$
\Omega=\max _{t=1, N_{a p}}\left(\prod_{k}^{n_{a}} \sigma_{k}\right)
$$

where $N_{a p}$ is the number of the existing positions and $n_{a}$ are the number of the actuators. 
(a)

$$
\begin{aligned}
& \begin{array}{c|c|c|c|c|c|c|}
\hline 1 & 0 & 1 & \cdots & 0 & 1 & 0 \\
\hline \hline 0 & 0 & 1 & \cdots & 0 & 1 & 1 \\
\hline \multicolumn{5}{c|}{\vdots} \\
\hline
\end{array} \\
& \begin{array}{|l|l|l|l|l|l|l|}
\hline 1 & 0 & 0 & \cdots & 1 & 1 & 0 \\
\hline
\end{array}
\end{aligned}
$$

$$
\begin{array}{|l|l|l|l|l|l|l|l|l|l|l|l|}
\hline 1 & 0 & 1 & \cdots & 0 & 1 & 0 \\
\hline 0 & 0 & 1 & \cdots & 0 & 1 & 1 \\
\hline
\end{array}, \begin{array}{lllllll|l|l|l|l|}
\hline & 0 & 1 & \cdots & 0 & 1 & 1 \\
\hline
\end{array}
$$

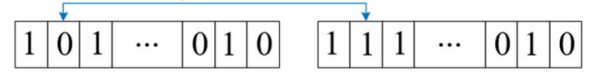

Figure 4. (a) Binary chromosome, (b) crossover, (c) mutation.

Norm of SVD. To find the optimal locations of the actuators, the norm of SVD has been maximized ${ }^{1}$. The objective function is indicated as

$$
\Omega=\max _{t=1, N_{a p}}\left(\max _{k=1, n_{a}} \sigma_{k}\right)
$$

The modified control matrix and singular value decomposition (MCSVD). The rows and columns of the control matrix $B_{c}$ demonstrate the states of the system and the number of actuators as inputs that are given to the system, respectively. In the MCSVD approach, the singular values of the column control matrix are taken as the fitness function because of increasing closed-loop average $\mathrm{dB}$ gain, and also optimal positions of the sensor and actuators are found by maximizing the fitness function ${ }^{1,25}$. The number of actuators on the plate are composed to create a large size actuator by collecting the columns of the control matrix to form a column matrix ${ }^{1,25}$. The singular value of the column matrix is dependent on locations which is provided by a combined patch (Eq. (44)) to form of a column matrix (Eq. (45)).

$$
\begin{gathered}
B_{c}=\left[\begin{array}{ccc}
0 & \cdots & 0 \\
\vdots & \cdots & \vdots \\
0 & \cdots & 0 \\
B_{11} & \cdots & B_{1 n_{a}} \\
\vdots & \cdots & \vdots \\
B_{n 1} & \cdots & B_{n n_{a}}
\end{array}\right]_{4 n \times n_{a}} \\
B_{c 1}=\left[\begin{array}{c}
0 \\
\vdots \\
0 \\
B_{11}+B_{12}+\cdots+B_{1 n_{a}} \\
\vdots \\
B_{n 1}+B_{n 2}+\cdots+B_{n n_{a}}
\end{array}\right]_{4 n \times 1}
\end{gathered}
$$

The singular value of the matrix $B_{c 1}$ is equal to the square roots of the eigenvalues of the matrix $B_{c 1} B_{c 1}^{T}$.

$$
\Omega=\max _{t=1, N_{a p}}\left(\sqrt{\left(\operatorname{eig}\left(\left[B_{c 1}\right]^{*}\left[B_{c 1}\right]^{T}\right)\right)}\right)
$$

The optimal location of piezoelectric patches are obtained by maximizing the Eq. (46) known as the controllability index that was calculated by maximizing the global force with binary-coded Genetic algorithm.

1-bit coding plate by genetic algorithm. 1-bit Coding plate is composed of only two kinds of unit cells with ' 0 ' and ' 1 ' response. When an actuator is not installed on a position, the coding state is ' 0 ' and otherwise, it is ' 1 '. Therefore, the chromosome is characterized by a binary demonstration of a combination of actuators. Figure 4 illustrates the binary chromosome, the crossover, and the punctual mutation. The binary code length is based on the number of elements of structures on the plate, which has been considered to be 100 .

Many strategies have been used to apply the reproduction process, but all involve "crossover" and "mutation". In a crossover, new chromosomes are produced by swapping pieces of parent's chromosomes. One or more digits in a chromosome are changed randomly in mutation ${ }^{1}$. In this way, crossover is used to explore the known areas of the search space by trial different combinations of digits, while mutation maintains variety in the population and so to explore new areas of the search space and prevent the process from getting trapped in a local optimal solution ${ }^{4}$. Moreover, ensuring that two individuals generated are not allowed having the same values in given domain for the present case with crossover and mutation. Therefore, a constraint has been set to get unique individuals. 
It is required to verify whether the individuals generated are valid after the genetic operations. In other words, it is significant to check whether all the binary representations gained after the crossover and the mutation follow the number of active elements which are between one and a maximum number desired. The binary representation sometimes is invalid because the number of actuators was not according to this maximum number (10 in this problem). So, this binary number must be replaced. If invalid individuals appear, it is essential to replace them to valid individuals. In the case of invalid individuals, these are changed by valid binary representations randomly selected. First, the values of each gene on each chromosome are summed with each other. If the sum is different from the total number of actuators mentioned, a valid representation is selected randomly. Thus, valid genes will always be obtained.

The GA process in this paper is given as follows:

1. The initial population is created randomly which the length of chromosome is equal to the total number of elements on the pate (i.e. 100).

2. A fitness value is computed for population members based on using a maximization problem instead of the minimization problem with the fitness $-\Omega$ according to Eq. (46). With a constraint which two gens of generated population are not allowed having same value and gens have absolute value.

3. The chromosomes are sorted according to their fitness value (controllability index).

4. Parents are selected, based on the highest fitness value.

5. Some individuals in the current population which have higher value of fitness are selected as elite to transfer to the next population.

6. Generate a new offspring by using the crossover on the selected parents (the crossover point being selected randomly).

7. Mutate one or two digits of the child's chromosome with certain probability.

8. Evaluate the fitness value for new population members, and find the best one.

9. Feasibility test: go to step 11 if there are infeasible individuals. Otherwise, go to step 10 .

10. Change the non-feasible individuals into feasible individuals and go to step 11.

11. If the predefined stop-condition is satisfied, the GA iteration is stopped, and returns the best solution in the current population.

12. Otherwise, go back to step 2. Put the previous result in the initial random population and repeat the process to obtain the global optimum.

The coding plate was founded by the MCSVD approach to suppress the first six modes. Figure 5 presents the flowchart of a GA for finding optimal number and placement of piezoelectric patches on the cantilever thick plate for suppressing the first six modes.

For the problem at hand, the ending condition of the GA is chosen to reach a certain number of iterations. For optimization, the configuration of GA in this work is considered as: crossover rate: 0.8 , Stopping criterion: when the largest cost of the chromosomes cannot be further increased in 50 generations.

Average closed-loop dB gain reduction. This method is applied to validate the optimal placements of piezoelectric patches obtained. Figures 6 and 7 show the open-loop (The control is performed without monitoring its output as there is no feedback) and closed-loop (The control is performed with sensors, composing a feedback that closes the loop) control.

The open-loop gain in $\mathrm{dB}$ is ${ }^{26}$ :

$$
G O_{k, t_{m}}=20 \log _{10}\left(\left|C_{c_{k}}\left(j \omega_{t_{m}} I-A_{s}\right)^{-1} B_{c_{k}}\right|\right)
$$

where $t_{m}$ represents the specified natural mode at the sensor number $k$ when a unite voltage used to the first actuator. $A_{s}, B_{c_{k}}$ and $C_{c_{k}}$ calculate by Eqs. (32) and (36).

By apply the sinusoidal unit voltage at a specified single actuator for all of the sensors and modes, the average open-loop $\mathrm{dB}$ gain is ${ }^{26}$ :

$$
M G O=\frac{1}{n_{m} n_{a}} \sum_{t_{m}=1}^{n_{m}} \sum_{k=1}^{n_{a}} G O_{k, t_{m}}
$$

The closed-loop state matrix is:

$$
A_{C_{s}}=A_{s}-B_{c_{k}} K_{k}
$$

The closed-loop dB gain $i$ is ${ }^{26}$ :

$$
G C_{k, t_{m}}=20 \log _{10}\left(\left|C_{c_{k}}\left(j \omega_{t_{m}} I-A_{C_{s}}\right)^{-1} B_{c_{k}}\right|\right)
$$

The mean average closed-loop dB gain for different values of linear quadratic matrices is ${ }^{26}$ : 


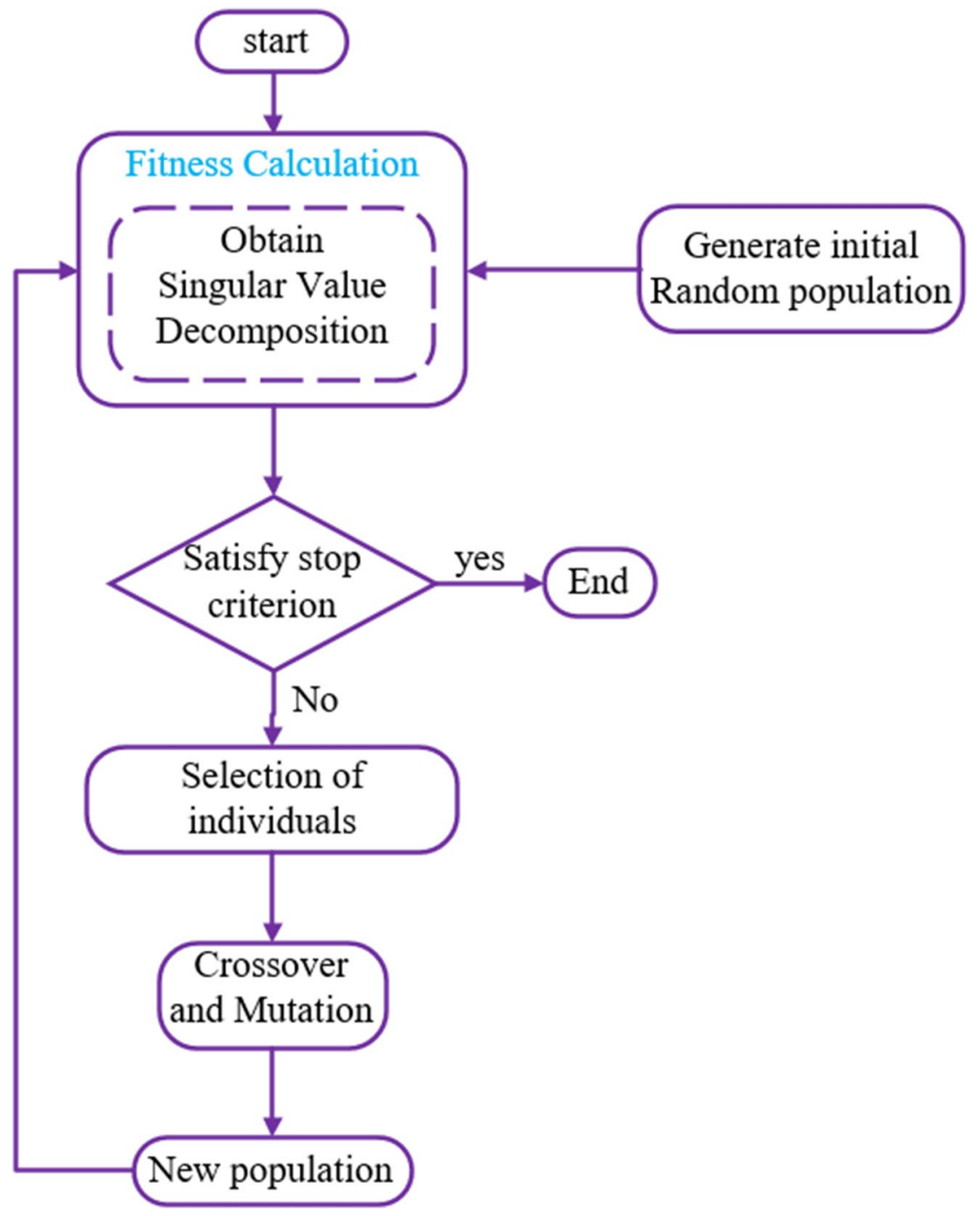

Figure 5. The flowchart of the genetic algorithm (GA).

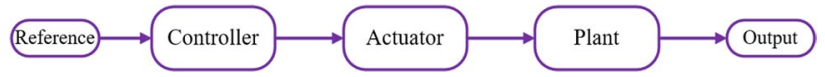

Figure 6. General open-loop control system.

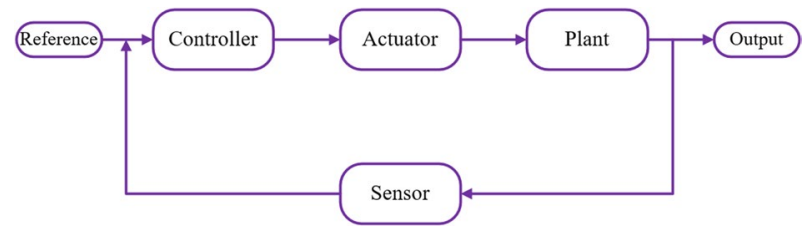

Figure 7. General closed-loop control system. 


\begin{tabular}{|l|l|l|}
\hline Parameter & PZT-5H & Plate \\
\hline $\mathrm{x}$-Length $[\mathrm{m}]$ & 0.1 & 1 \\
\hline $\mathrm{y}$-Length $[\mathrm{m}]$ & 0.05 & 0.5 \\
\hline Thickness $[\mathrm{m}]$ & 0.005 & 0.06 \\
\hline$\rho\left[\mathrm{kg} / \mathrm{m}^{3}\right]$ & - & 2770 \\
\hline $\mathrm{E}[\mathrm{Gpa}]$ & - & 70 \\
\hline$\nu[-]$ & - & 0.03 \\
\hline$\alpha_{\mathrm{M}}=\beta_{\mathrm{K}}$ & - & $5 \times 10^{-5}$ \\
\hline $\mathrm{C}_{11}=\mathrm{C}_{22}=\mathrm{C}_{33}, \mathrm{C}_{12}[\mathrm{GPa}]$ & $126.0,79.5$ & - \\
\hline $\mathrm{C}_{13}=\mathrm{C}_{23}, \mathrm{C}_{44}=\mathrm{C}_{55}=\mathrm{C}_{66}[\mathrm{GPa}]$ & $84.1,23.0$ & - \\
\hline$\chi_{11}=\chi_{22}, \chi_{33}[\mathrm{~F} / \mathrm{m}]$ & $1.503 \times 10^{-8}, 1.30 \times 10^{-8}$ & - \\
\hline $\mathrm{e}_{15}=\mathrm{e}_{24}, \mathrm{e}_{31}=\mathrm{e}_{32}, \mathrm{e}_{33}\left[\mathrm{C} / \mathrm{m}^{2}\right]$ & $17,-6.5,23.3$ & - \\
\hline
\end{tabular}

Table 1. Geometrical and physical data of the plate with the piezoelectric patches ${ }^{27}$.

\begin{tabular}{|l|c|l|}
\hline Natural frequencies $\mathbf{( H z )}$ & MATLAB & ABAQUS \\
\hline Frequency 1 & 193.1245 & 189.18 \\
\hline Frequency 2 & 315.1004 & 308.61 \\
\hline Frequency 3 & 598.7914 & 576.30 \\
\hline Frequency 4 & 1147.72 & 1040.3 \\
\hline Frequency 5 & 1169.909 & 1125.2 \\
\hline Frequency 6 & 1304.712 & 1277.3 \\
\hline
\end{tabular}

Table 2. The first six natural frequencies of the FDM model obtained through MATLAB codes, and ABAQUS.
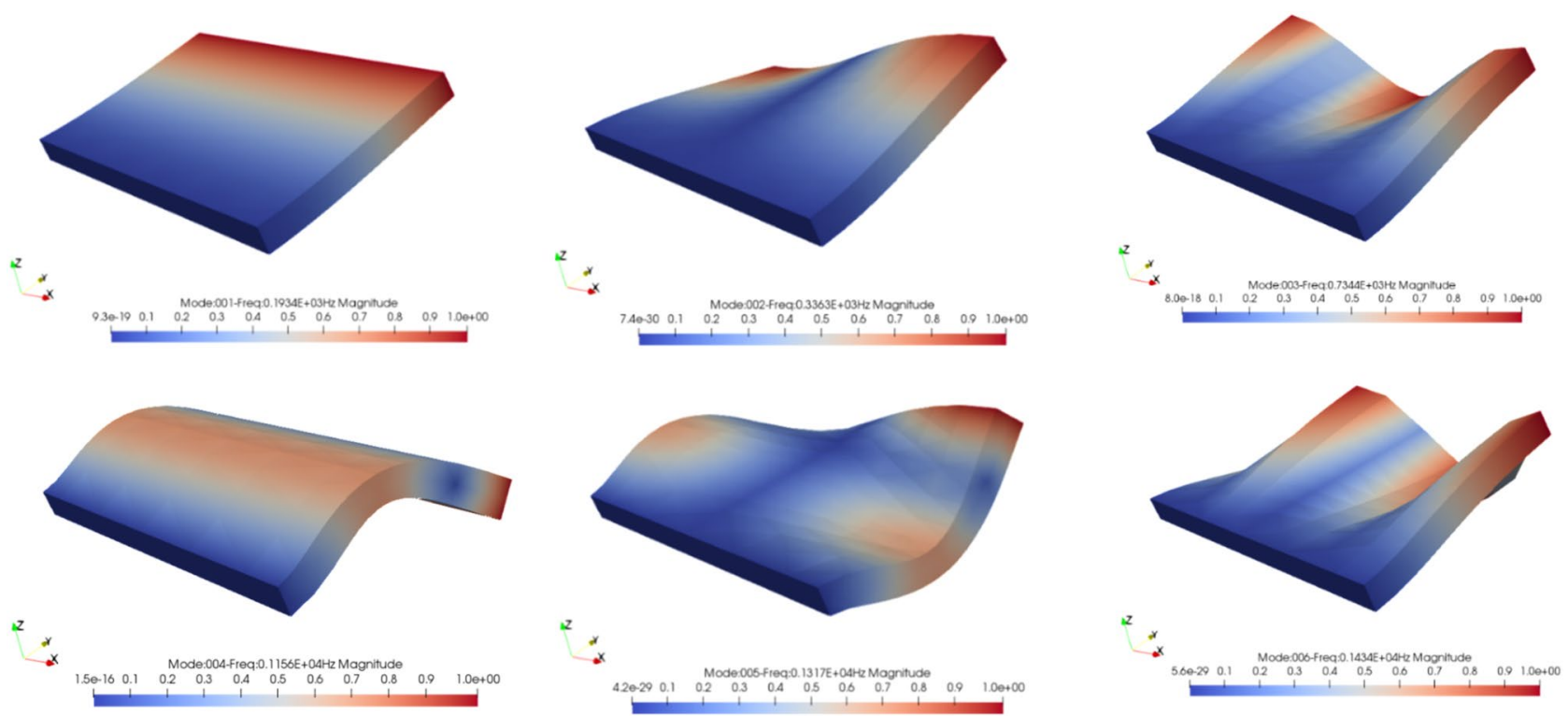

Figure 8. The first six modal shapes of a thick cantilever plate by FORTRAN. 


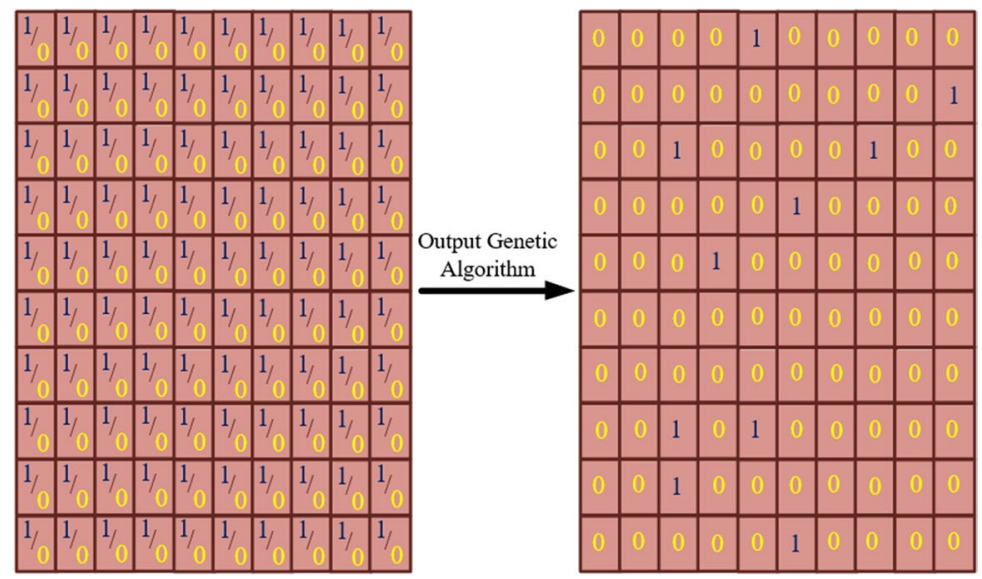

Figure 9. The piezoelectric output array of genetic algorithm.

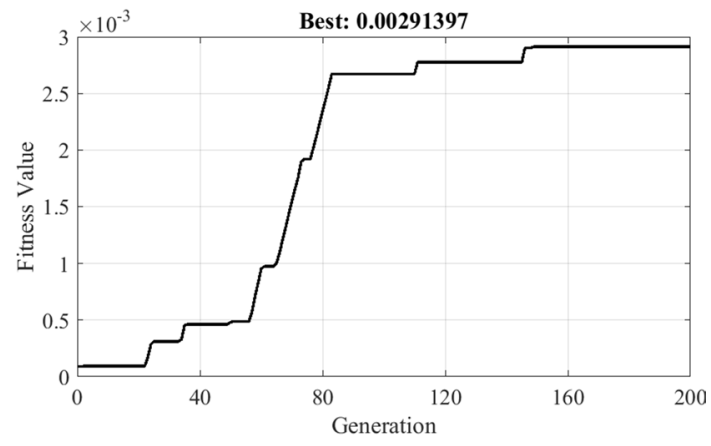

Figure 10. The convergence of the fitness value.

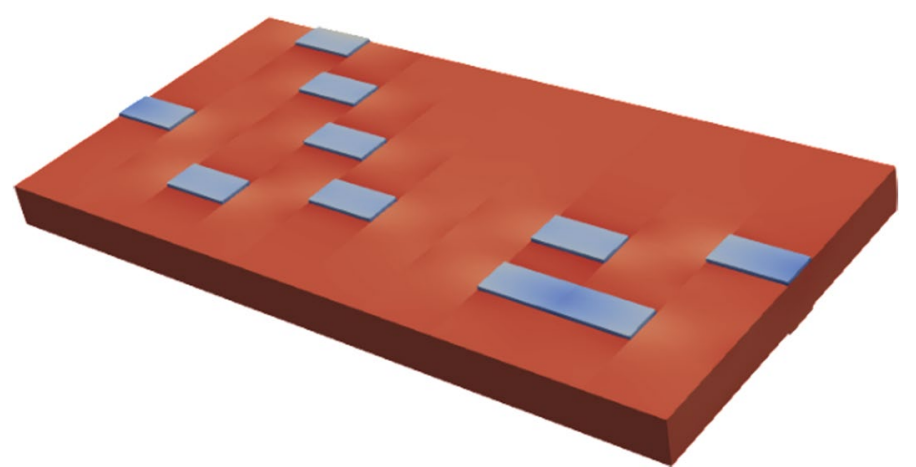

Figure 11. Ten piezoelectric patches on a cantilever thick plate by FORTRAN. 

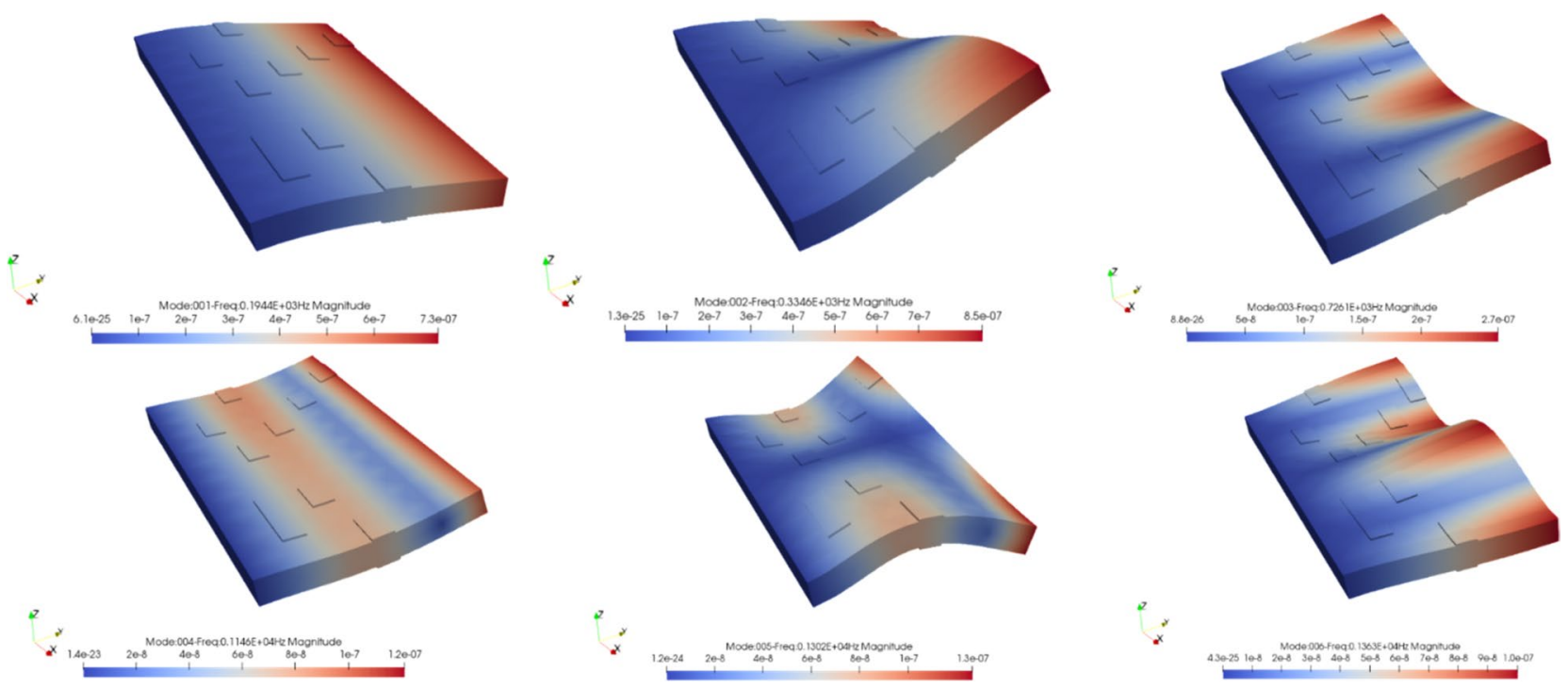

Figure12. The first six modal shapes of the plate by FORTRAN.

\begin{tabular}{|l|r|r|r|r|l|l|l|l|l|l|}
\hline Ten S/A pairs & \multicolumn{10}{|c|}{ Element number based on Fig. 13 } \\
\hline Present study (Eq. (46)) & \multicolumn{1}{|c|}{23} & 28 & 29 & 35 & 41 & 48 & 54 & 60 & 73 & 92 \\
\hline Present study (Eq. (43)) & 28 & 33 & 35 & 37 & 43 & 46 & 48 & 54 & 75 & 77 \\
\hline Ref. $^{18}$ & 1 & 2 & 5 & 9 & 11 & 36 & 45 & 56 & 95 & 96 \\
\hline Random & 23 & 28 & 57 & 63 & 64 & 67 & 68 & 75 & 76 & 90 \\
\hline
\end{tabular}

Table 3. Location of piezoelectric patches in different cases.

$$
M G C=\left.\frac{1}{3 n_{m} n_{a}}\left|\sum_{q=1}^{3} \sum_{t_{m}=1}^{n_{m}} \sum_{k=1}^{n_{a}} G C_{k, t_{m}}\right|\right|_{\begin{array}{c}
R=1 \\
Q_{k}=10^{6}, 10^{7}, 10^{8}
\end{array}}
$$

The average closed-loop $\mathrm{dB}$ gain reduction is defined as:

$$
M G R=|M G C-M G O|
$$

\section{Results and discussion}

The properties and dimensions of the cantilever thick plate and piezoelectric patches are given in Table 1.

The first resonance modes of the structure were obtained by applying the eigenvalue algorithm to the FDM model. The frequencies and shapes associated with some of these are presented in Table 2 and Fig. 8. It should be noted that the reason for the difference between the frequencies obtained between MATLAB and Abacus software in Table 2 is the difference between the number of elements used for modeling. Figure 8 illustrates that the shapes of the first six modes are symmetrically distributed about the plane of symmetry of the plate, which is perpendicular to its fixed end.

The cantilever rectangular thick plate was discretized in $10 \times 10$ quadrangular elements after optimization by GA which shows ten patches from the list of binary representations (possible solutions between one to ten patches on the plate) after terminating the conditions (Fig. 9). Moreover, the goal is not to find symmetrical places. The convergence of the fitness function for the case of cantilever thick plate indicates in Fig. 10.

The ParaView representation of the thick plate with piezoelectric patches can be found in Fig. 11. Discrete piezoelectric pairs (actuator/sensor) give high actuating and sensing effects if they are appropriately located.

Figure 12 shows the first six mode shapes of the thick plate with ten piezoelectric pairs, which are symmetrically distributed like Fig. 8 . 


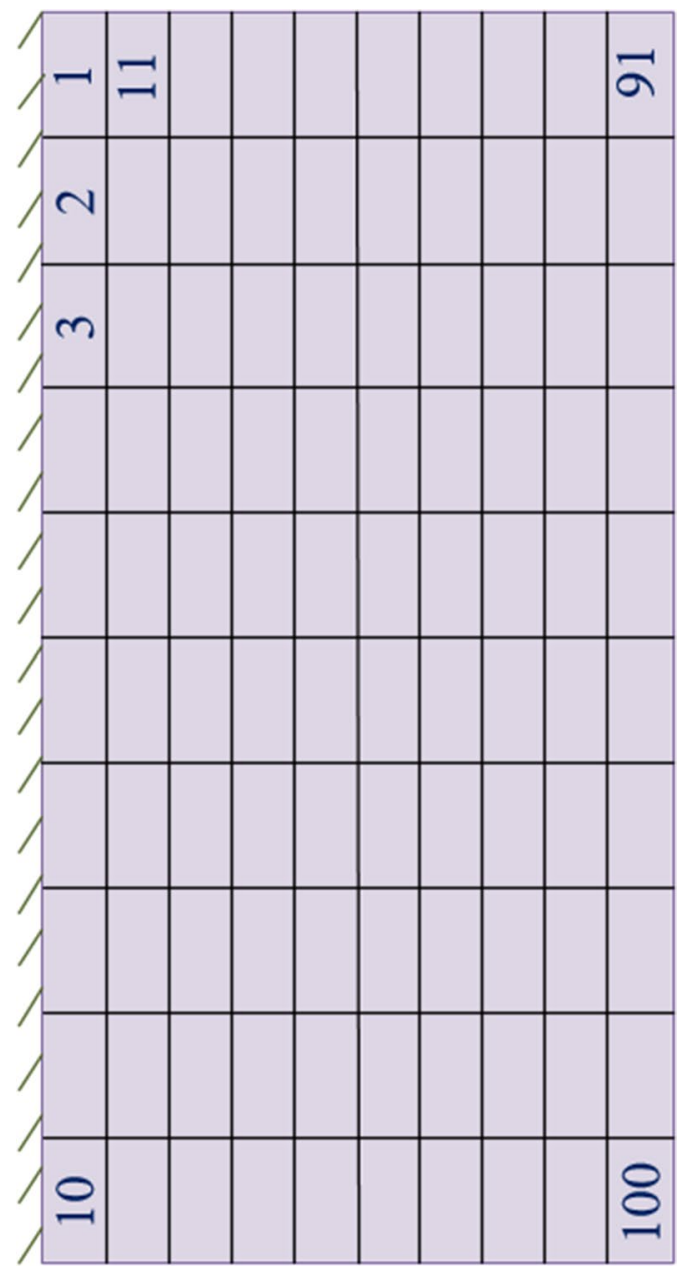

Figure 13. Cantilever smart plate having 100 elements.

\begin{tabular}{|l|c|l|l|l|}
\hline \multirow{2}{*}{ Ten S/A pairs } & \multicolumn{4}{|c|}{ Closed-loop dB gain reduction } \\
\cline { 2 - 5 } & $\mathbf{Q = 1 0 ^ { 5 }}$ & $\mathbf{Q = 1 0 ^ { \mathbf { 6 } }}$ & $\mathbf{Q = 1 0 ^ { 7 }}$ & $\mathbf{Q = 1 0 ^ { \mathbf { 8 } }}$ \\
\hline Present study (Eq. (46)) & 11.22 & 13.02 & 15.12 & 18.00 \\
\hline Present study (Eq. (43)) & 8.87 & 10.53 & 12.56 & 15.32 \\
\hline Ref. $^{18}$ & 8.06 & 9.82 & 11.84 & 14.67 \\
\hline Random & 7.96 & 9.45 & 11.13 & 13.54 \\
\hline
\end{tabular}

Table 4. Closed-loop average $\mathrm{dB}$ gain reduction for the cantilever thick bonded with ten piezoelectric sensor/ actuator pairs.

\begin{tabular}{|l|l|l|l|l|}
\hline \multirow{2}{*}{ Ten S/A pairs } & \multicolumn{4}{|c|}{ Percentage reduction improvement } \\
\cline { 2 - 5 } & $\mathbf{Q = 1 0 ^ { 5 }}$ & $\mathbf{Q = 1 0 ^ { \mathbf { 6 } }}$ & $\mathbf{Q = 1 0 ^ { \mathbf { 7 } }}$ & $\mathbf{Q = 1 0 ^ { \mathbf { 8 } }}$ \\
\hline Present study (Eq. (43)) & $26.49 \%$ & $23.65 \%$ & $20.38 \%$ & $17.49 \%$ \\
\hline Ref. $^{18}$ & $39.21 \%$ & $32.26 \%$ & $27.70 \%$ & $22.70 \%$ \\
\hline Random & $40.95 \%$ & $37.78 \%$ & $35.85 \%$ & $32.94 \%$ \\
\hline
\end{tabular}

Table 5. Percentage reduction improvement for present study (Eq. (46)) than each case. 


\begin{tabular}{|c|c|c|c|c|c|c|c|c|c|c|}
\hline \multirow[b]{2}{*}{ Controlled the first six modes } & \multicolumn{10}{|c|}{ Number of patches } \\
\hline & 1 & 2 & 3 & 4 & 5 & 6 & 7 & 8 & 9 & 10 \\
\hline System number & System 1 & System 2 & System 3 & System 4 & System 5 & System 6 & System 7 & System 8 & System 9 & System 10 \\
\hline Element number & 23 & 28 & 29 & 35 & 41 & 48 & 54 & 60 & 73 & 92 \\
\hline
\end{tabular}

Table 6. Optimal placement of piezoelectric pairs installed to the rectangular thick plate.

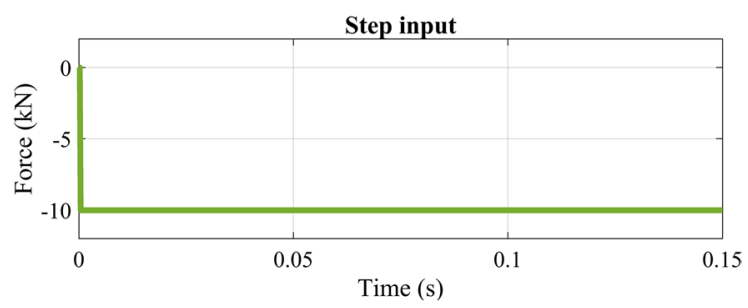

Figure 14. Step input force in $t=0.0002 \mathrm{~s}$ at $(x, y, z)=(0,0.269375 \mathrm{~m}, 0.03 \mathrm{~m})$.

The location of piezoelectric patches for each case are given in Table 3 based on the element number on the thick plate, which are shown in Fig. 13.

The state-space matrices are determined for the cantilever thick plate bonded with piezoelectric actuator and sensor pairs in optimal placement and optimal number based on Eq. (46). Then the optimal feedback control gain matrix calculates by using optimal linear quadratic control with weighted matrices $\mathrm{R}=1$ and $\mathrm{Q}=10^{5}, 10^{6}, 10^{7}, 10^{8}$. The closed-loop average vibration reduction using an optimal linear quadratic control scheme for the cantilever thick plate with ten sensor/ actuator pairs optimally located was compared with that of optimal piezoelectric configurations in the previously published study ${ }^{18}$, the result of the thin plate has been used on the thick plate), based on Eq. (43) and random piezoelectric configurations (Selection 10 numbers between 1 and 100 in randomly by MATLAB software), and the final results are reported in Table 4 . It is clear that the present global optimal ten piezoelectric pairs (based on Eq. (46)) give an average improved vibration reduction between 17.49 and $40.95 \%$ compared to other sensor/actuator pairs configurations (see Table 5). Table 5 shows that percentage reduction improvement in the MCSVD approach is more than this value of the norm of SVD. This is also true for the random selection of piezoelectric configurations. In addition, using optimal locations for piezoelectric patches obtained on thin plates is not recommended for thick plates because there is less percentage reduction improvement than the value of using optimal placement based on thick plate (Eqs. (43) and (46)). An increase in the weighted matrix $Q$ to a value is higher than $10^{8}$ causes noise and locations divergence of the poles of the closed-loop system on the s-plane.

The systems related to the sensor-actuator pairs are introduced in Table 6 . In this system, each actuator will be responsible to minimize only the sensor voltage right below it. Other techniques could be used aiming at the minimization of both sensor voltages simultaneously for each controller, but the results would be similar. The main reason for this result is that the control applied by each actuator affects its nearest sensor much more than the others.

The vertical motion of the point where the force was applied (middle point) for step disturbance (Fig. 14) is represented in Fig. 15.

The maximum transverse displacement value for all systems in Fig. 15 is about $2 \times 10^{-1}$ for impulse input. By using only 10 piezoelectric patches, the amplitude reduction is achieved over all 6 modes. The above results indicate that using an array of appropriately positioned discrete actuators is necessary to provide good controllability of the structure. The increased effectiveness is attributed to locating actuators in the best possible positions for control of all 6 structural modes. Therefore, the effect of optimal location of piezoelectric patches in suppressing displacement can be observed.

\section{Conclusions}

In this paper, a binary-coded genetic algorithm which modified by adding feasibility test in the mutation step was applied to optimize the location and the number of piezoelectric patches on the cantilever thick plate in order to suppress the vibration. The objective function was obtained by modifying the control matrix and SVD (MCSVD). It is argued that the optimal positions and numbers calculated by the present method have raised the closed-loop average $\mathrm{dB}$ reduction gain compared with using random placement, norm of SVD, and the result of optimal placement on thin-plate. It has been found that the present optimal placement gives a higher average $\mathrm{dB}$ gain reduction of $26.49 \%, 23.65 \%, 20.38 \%$, and $17.49 \%$ than the other cases at $\mathrm{R}=1$ and $\mathrm{Q}=10^{5}, 10^{6}, 10^{7}, 10^{8}$, respectively. The present objective function obtains a higher vibration reduction.

Future research in the respect of active vibration control includes number of topics dealing with the optimization of sensors and actuators, the type of structure considered and the theoretical and experimental application of different control schemes to suppress vibration. The following topics would be prolific areas for future investigation. 

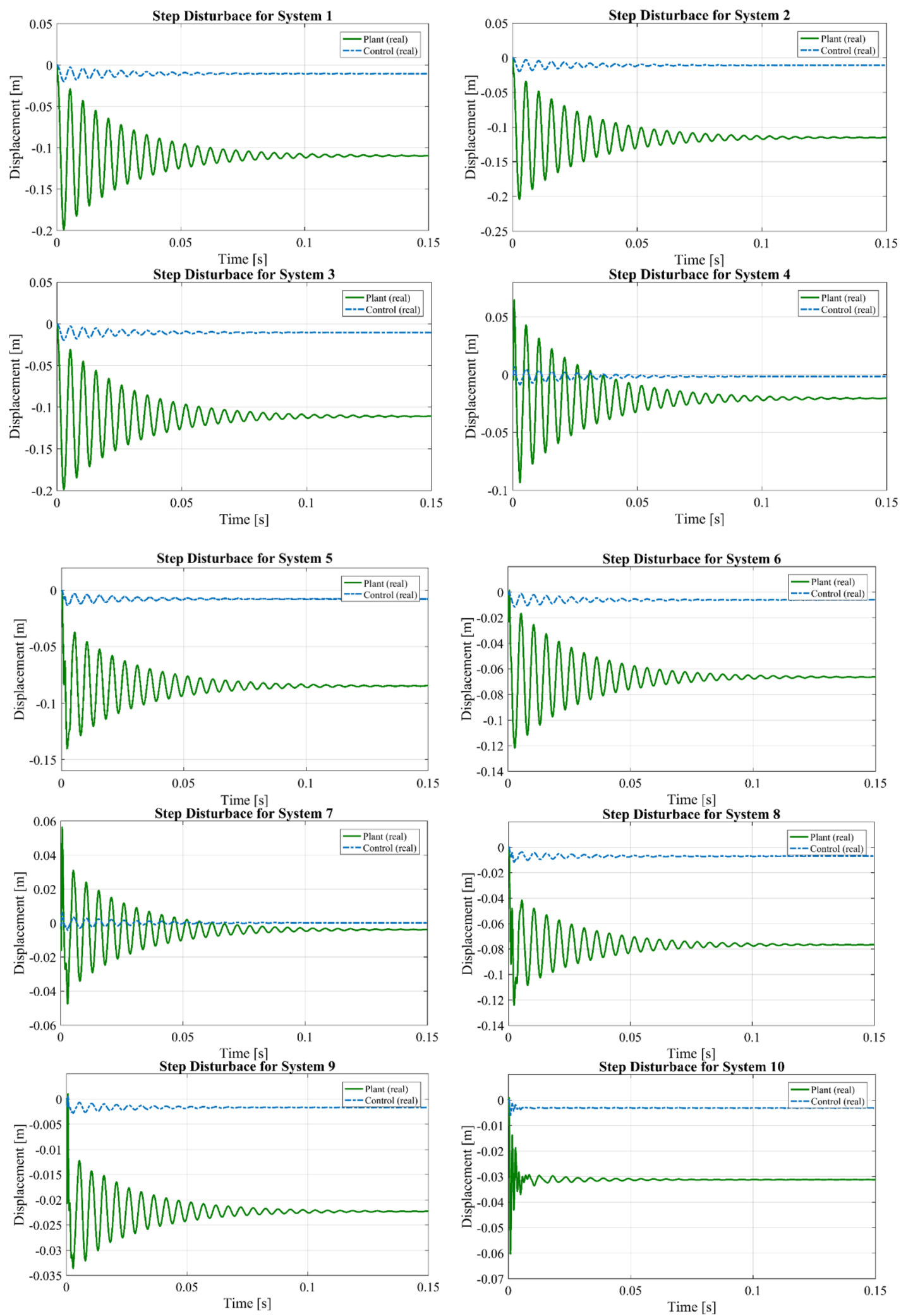

Figure 15. Step input: vertical displacement of the middle point with PID controller for all systems. 
1. Investigation of active vibration reduction in stiffened plates, with and without symmetry, so as to implement the placement strategies and objective functions used in this study. Shells and plates stiffened by beams have been used intensively in the manufacturing of mechanical structures with high specific strength.

2. Investigation of reducing the search space in the optimization problem for active vibration control in flexible structures. A half-chromosome technique can be extended based on the finding that the global optimal configuration of actuators and sensors followed the structural dynamic axes of symmetry. The use of this technique greatly reduced the search space of the optimization problem and holds great potential for finding global optimal solution to large-scale mechanical structures after a small number of generations.

3. Further improvements to the effectiveness of active vibration control by optimal placement of sensors and actuators might be investigated using optimal proportional differential, LQR and fuzzy neural control schemes.

Received: 6 April 2021; Accepted: 22 June 2021

Published online: 02 July 2021

\section{References}

1. Chhabra, D., Bhushan, G. \& Chandna, P. Optimal placement of piezoelectric actuators on plate structures for active vibration control via modified control matrix and singular value decomposition approach using modified heuristic genetic algorithm. Mech. Adv. Mater. Struct. 23, 272-280 (2016).

2. Jia, S., Jia, Y., Xu, S. \& Hu, Q. Optimal placement of sensors and actuators for gyroelastic body using genetic algorithms. J. Aircr. 53, 2472-2488 (2016).

3. Padoin, E., Fonseca, J. S. O., Perondi, E. A. \& Menuzzi, O. Optimal placement of piezoelectric macro fiber composite patches on composite plates for vibration suppression. Lat. Am. J. Solids Struct. 12, 925-947 (2015).

4. Bruant, I., Gallimard, L. \& Nikoukar, S. Optimal piezoelectric actuator and sensor location for active vibration control, using genetic algorithm. J. Sound Vib. 329, 1615-1635 (2010).

5. Chakraborty, D., Rathore, P. K. \& Roy, T. Optimal actuators locations in smart fiber reinforced polymer structures using genetic algorithm. Appl. Mech. Mater. 110-116, 1278-1283 (2012).

6. Biglar, M., Gromada, M., Stachowicz, F. \& Trzepieciński, T. Optimal configuration of piezoelectric sensors and actuators for active vibration control of a plate using a genetic algorithm. Acta Mech. 226, 3451-3462 (2015).

7. Loghmani, A., Danesh, M. \& Keshmiri, M. Modal structural acoustic sensing with minimum number of optimally placed piezoelectric sensors. J. Sound Vib. 363, 345-358 (2015).

8. Ferrari, G. \& Amabili, M. Active vibration control of a sandwich plate by non-collocated positive position feedback. J. Sound Vib. 342, 44-56 (2015).

9. Soubhia, A. L. \& Serpa, A. L. Discrete optimization for actuator and sensor positioning for vibration control using genetic algorithms. JVC/J. Vib. Control 24, 4050-4064 (2018).

10. Daraji, A. H. \& Hale, J. M. Active vibration reduction of a flexible structure bonded with optimised piezoelectric pairs using half and quarter chromosomes in genetic algorithms. J. Phys. Conf. Ser. 382, 012039 (2012).

11. Daraji, A. H. \& Hale, J. M. Conditional filter for reduction of the search space in genetic algorithm optimisation of sensor and actuator location in active vibration control. ICSV. 19. 2, 1199-1211 (2012).

12. Gomes, G. F. et al. Sensor placement optimization applied to laminated composite plates under vibration. Struct. Multidiscip. Optim. 58, 2099-2118 (2018).

13. Błachowski, B. et al. Convex relaxation for efficient sensor layout optimization in large-scale structures subjected to moving loads. Comput. Civ. Infrastruct. Eng. 35, 1085-1100 (2020).

14. Kammer, D. C. \& Tinker, M. L. Optimal placement of triaxial accelerometers for modal vibration tests. Mech. Syst. Signal Process. 18, 29-41 (2004).

15. Guo, H., Zhuang, X. \& Rabczuk, T. A deep collocation method for the bending analysis of Kirchhoff plate. Comput. Mater. Contin. 59, 433-456 (2019).

16. Samaniego, E. et al. An energy approach to the solution of partial differential equations in computational mechanics via machine learning: concepts, implementation and applications. Comput. Methods Appl. Mech. Eng. 362, 112790 (2020).

17. Wu, R. et al. Free and forced vibrations of SC-cut quartz crystal rectangular plates with the first-order Mindlin plate equations. Ultrasonics 73, 96-106 (2017).

18. Nanthakumar, S. S. et al. Detection of material interfaces using a regularized level set method in piezoelectric structures. Inverse Probl. Sci. Eng. 24, 153-176 (2016).

19. Senjanović, I., Vladimir, N. \& Tomić, M. An advanced theory of moderately thick plate vibrations. J. Sound Vib. 332, 1868-1880 (2013).

20. Senjanović, I., Vladimir, N., Cho, D. S. \& Choi, T. M. Vibration analysis of thick plates: analytical and numerical approaches. OMAE. 45424 (2014).

21. Kumar, K. R. \& Narayanan, S. The optimal location of piezoelectric actuators and sensors for vibration control of plates. Smart Mater. Struct. 16, 2680-2691 (2007).

22. Abdeljaber, O., Avci, O. \& Inman, D. J. Active vibration control of flexible cantilever plates using piezoelectric materials and artificial neural networks. J. Sound Vib. 363, 33-53 (2015).

23. Qiu, Z. C., Zhang, X. M., Wu, H. X. \& Zhang, H. H. Optimal placement and active vibration control for piezoelectric smart flexible cantilever plate. J. Sound Vib. 301, 521-543 (2007).

24. Wang, Q. \& Wang, C. M. A controllability index for optimal design of piezoelectric actuators in vibration control of beam structures. J. Sound Vib. 242, 507-518 (2001).

25. Vashist, S. K. \& Chhabra, D. Optimal placement of piezoelectric actuators on plate structures for active vibration control using genetic algorithm. Act. Passiv. Smart Struct. Integr. Syst. 9057, 71 (2014).

26. Daraji, A. H. \& Hale, J. M. Reduction of structural weight, costs and complexity of a control system in the active vibration reduction of flexible structures. Smart Mater. Struct. 23, 095013 (2014).

27. Hale, J. M. \& Daraji, A. H. Optimal placement of sensors and actuators for active vibration reduction of a flexible structure using a genetic algorithm based on modified H_infinity. J. Phys. Conf. Ser. 382, 012036 (2012). 


\section{Author contributions}

A.N., M.M. and E.A.Y. developed the concept. A.N. did the simulations. A.N. wrote the manuscript based on the suggestions from all authors. A.N. prepared all figures (A.N. drew the Figs. 1, 2, 3, 4, 5, 6 and 7, 9, and 13 by Microsoft Visio). M.M. and E.A.Y. reviewed the manuscript. All authors were involved in the discussions.

\section{Funding}

This research did not receive any specific grant from funding agencies in the public, commercial, or not-forprofit sectors.

\section{Competing interests}

The authors declare no competing interests.

\section{Additional information}

Supplementary Information The online version contains supplementary material available at https://doi.org/ 10.1038/s41598-021-93136-5.

Correspondence and requests for materials should be addressed to A.N.

Reprints and permissions information is available at www.nature.com/reprints.

Publisher's note Springer Nature remains neutral with regard to jurisdictional claims in published maps and institutional affiliations.

(c) (i) Open Access This article is licensed under a Creative Commons Attribution 4.0 International License, which permits use, sharing, adaptation, distribution and reproduction in any medium or format, as long as you give appropriate credit to the original author(s) and the source, provide a link to the Creative Commons licence, and indicate if changes were made. The images or other third party material in this article are included in the article's Creative Commons licence, unless indicated otherwise in a credit line to the material. If material is not included in the article's Creative Commons licence and your intended use is not permitted by statutory regulation or exceeds the permitted use, you will need to obtain permission directly from the copyright holder. To view a copy of this licence, visit http://creativecommons.org/licenses/by/4.0/.

(C) The Author(s) 2021 